\title{
Presentation of Hyperelliptic Periodic Monodromies and Splitting Families
}

\section{Mizuho ISHIZAKA}

\author{
Mizuho Ishizaka \\ Department of Mathematics \\ School of Science and Engineering \\ Waseda University \\ 3-4-1, Ohkubo Shinjuku \\ Tokyo 1698050 - Japan \\ ishizakamizuho@yahoo.co.jp
}

Received: November 14, 2006

Accepted: April 23, 2007

\section{ABSTRACT}

Let $\Sigma_{g}$ be an oriented connected real two dimensional manifold of genus $g$ without boundary. For periodic homeomorphisms of $\Sigma_{g}$ that commute with a hyperelliptic involution, we give a method to obtain their presentations by Dehn twists.

Key words: mapping class group, degeneration.

2000 Mathematics Subject Classification: 14D06, 14H45, 14H15, 57M99, 30F99.

\section{Introduction}

Let $\Sigma_{g}$ be an oriented connected real two dimensional manifold of genus $g$ without boundary and $\mathcal{M}_{g}$ the mapping class group of genus $g$. We denote the conjugacy class of an element $\phi$ in $\mathcal{M}_{g}$ by $[\phi]$. An element $\phi$ of $\mathcal{M}_{g}$ is said to be periodic when there exists a positive integer $n$ such that $\phi^{n}=\mathrm{id}_{\Sigma_{g}}$, where $\mathrm{id}_{\Sigma_{g}}$ is the identity of $\mathcal{M}_{g}$. The smallest such integer $n$ is called the period of $\phi$. An involution $I$ of $\Sigma_{g}$ with $2 g+2$ fixed points is called a hyperelliptic involution. An element $\phi$ is said to be hyperelliptic if $\phi$ commutes with a hyperelliptic involution $I$. The conjugacy class of a hyperelliptic element is also said to be hyperelliptic.

As is well-known ([11]), any element of $\mathcal{M}_{g}$ can be written as a product of Dehn twists. For some elements of $\mathcal{M}_{g}$, we know the presentation of them by Dehn twists. 
For example, Birman and Hilden [2] obtained a presentation of the hyperelliptic involution by Dehn twists. Matsumoto [12] obtained a presentation of a certain involution in $\mathcal{M}_{2}$. Using the similar method of Matsumoto, Ito [8] obtained again the same presentation of the hyperelliptic involution obtained by Birman and Hilden.

However, for a given element $\phi \in \mathcal{M}_{g}$, there is no method to obtain a presentation of $\phi$ by Dehn twists, in general. Thus the problem to find such a method seems to be interesting.

In this paper, in the case where $\phi$ is hyperelliptic and periodic, we solve this problem modulo the conjugacy equivalence in the following way: If $\phi$ is hyperelliptic and periodic, the conjugacy class $[\phi]$ of $\phi$ can be realized as the topological monodromy of a degeneration whose general fibers are hyperelliptic curves (see [6, Remark 1.6]). Such degenerations are classified into six types ([6, Theorem 1.5]). By combining this results and the cyclic base change method, we reduce the problem into obtaining presentations of the three elements $\phi_{j}(j=1,2,3)$ in section 1 .

For each $\phi_{j}$, we obtained its presentation using "the splitting deformation." Roughly speaking, for the degeneration $f: S \rightarrow \Delta$ whose monodromy is $\left[\phi_{j}\right]$, we construct "a relative deformation" $\left\{f_{s}: S_{s} \rightarrow \Delta_{s}\right\}_{s \in \Delta}$ of $f$ such that $f_{s}(s \neq 0)$ is a Lefschetz fibration. Since the monodromy around the Lefschetz fiber is a Dehn twist along the vanishing cycle ([3] or [9]), we analyze the positions of the vanishing cycles and also the composition rule of these local monodromies around the Lefschetz fibers of $f_{s}$. Finally, we express explicitly $\left[\phi_{j}\right]$ as a product of Dehn twists whose center curves belong to the Birman-Hilden base. (See more precise definitions and the strategy in section 2.)

In section 1, first, we give the precise definitions of the degeneration of curves and its monodromy. Next, we review hyperelliptic degenerations and their monodromies.

In section 2 , we give the definition of splitting family of a degeneration and construct the splitting families of degenerations with monodromies $\left[\phi_{j}\right](j=1,2,3)$. Then, for each $\left[\phi_{j}\right]$, we give a presentation by Dehn twists.

In section 3 , using the results in section 2 , we introduce the method to obtain presentations for hyperelliptic periodic elements. At the end of section 3 , we add the list of periodic monodromies in the case where $g=2,3$.

\section{Review of hyperelliptic monodromy}

Let $f: S \rightarrow \Delta$ be a proper flat morphism from a two dimensional complex manifold $S$ to a small disk $\Delta:=\{t \in \mathbf{C}|| t \mid<\varepsilon\}$. If all but a finite number of fibers of $f$ are nonsingular algebraic curves of genus $g \geq 2$, we call a triple $(f, S, \Delta)$ a family of curves of genus $g$. If $f^{-1}(P)$ is singular, we call $P$ a critical point of $f$. Especially, we call a family of curves with only one critical point a degeneration of curves. We call a degeneration whose nonsingular fibers are hyperelliptic a hyperelliptic degeneration. For a degeneration $(f, S, \Delta)$, we can determine the topological monodromy as the conjugacy class in $\mathcal{M}_{g}$ as follows (see [13]): Let $l:[0,2 \pi] \rightarrow \Delta$ be a simple closed 
curve on $\Delta$ rounding the critical point with counter clockwise orientation. Fixing a homeomorphism $\phi_{0}^{l}: \Sigma_{g} \rightarrow f^{-1}(l(0))$, we can naturally define $\phi_{\theta}^{l}: \Sigma_{g} \rightarrow f^{-1}(l(\theta))$ continuously along $l$. Then we see that a monodromy homeomorphism $\phi_{f}^{l}:=\left(\phi_{0}^{l}\right)^{-1} \circ$ $\phi_{2 \pi}^{l}$ along $l$ is an orientation preserving homeomorphism. The conjugacy class $\left[\phi_{f}^{l}\right]$ of the isotopy class of $\phi_{f}^{l}$ can be uniquely determined and we call it the topological monodromy (the monodromy, for short) of $(f, S, \Delta)$. If we set $\phi_{0}^{l}:=\operatorname{id}_{f^{-1}(l(0))}, \phi_{f}^{l}$ is a homeomorphism of $f^{-1}(l(0))$. In this case, we call $f^{-1}(l(0))$ a reference fiber.

By a suitable coordinate change of $\Delta$, we may assume that $\Delta:=\{t \in \mathbf{C}|| t \mid<2\}$. Let $\left(X_{0}: X_{1}\right)$ be a homogeneous coordinates of $\mathbf{P}^{1}$. Let $\mathrm{pr}_{1}$ and $\mathrm{pr}_{2}$ be the first and the second projections of $\mathbf{P}^{1} \times \Delta$, respectively. Set $\Gamma_{t}:=\operatorname{pr}_{2}^{-1}(t)(t \in \Delta)$. Let $D$ be a positive reduced divisor on $\mathbf{P}^{1} \times \Delta$ whose defining equation is $F\left(X_{0}: X_{1}, t\right)=0$. Assume that $F\left(X_{0}: X_{1}, t\right)$ is homogeneous polynomial of degree $2 g+2(g \geq 2)$ with respect to $\left(X_{0}: X_{1}\right)$ and the equation $F\left(X_{0}: X_{1}, t\right)=0$ has $2 g+2$ distinct roots for each $t \in \Delta \backslash\{0\}$. We denote the line bundle associated to $D$ by $[D]$. Since $\Gamma_{t} D$ is even, there exists a line bundle $L$ satisfying that $L^{\otimes 2} \simeq[D]$ and we can construct the divisor $S$ on the total space $V(L)$ of the line bundle $L$ so that the restriction of the natural projection $\Pi: S \rightarrow \mathbf{P}^{1} \times \Delta$ induces a finite morphism of degree two branched

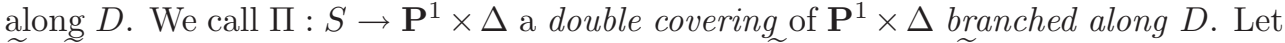
$\widetilde{\Pi}: \widetilde{S} \rightarrow S$ be a resolution of singularities of $S$. Set $\widetilde{f}:=\operatorname{pr}_{2} \circ \Pi \circ \widetilde{\Pi}$ and $f:=\operatorname{pr}_{2} \circ \Pi$, for short. From the conditions of $D$, we see that $(\widetilde{f}, \widetilde{S}, \Delta)$ is a hyperelliptic degeneration of genus $g$.

Though $S$ is not a manifold in general, we can also define the monodromy of $f: S \rightarrow \Delta$ by the same way because its singular points are always on its singular fiber. Moreover, we see that the monodromy of $f: S \rightarrow \Delta$ is equal to that of $f: \widetilde{S} \rightarrow \Delta$ from the definition of the monodromy. The monodromy of a hyperelliptic degeneration constructed as above is induced from "the monodromy of $2 g+2$ pointed $\mathbf{P}^{1}$ bundle $\mathbf{P}^{1} \times \Delta \rightarrow \Delta$ " as follows; Let $l:[0,2 \pi] \rightarrow \Delta$ be a simple closed curve rounding the critical point with counter clockwise orientation on $\Delta$ and $a_{i}^{\theta}(i=1, \ldots, 2 g+2)$ the roots of the equation of $F\left(X_{0}: X_{1}, l(\theta)\right)=0$. Set $R:=\left\{\left(X_{0}: X_{1}, l(\theta)\right) \in \mathbf{P}^{1} \times \Delta \mid\right.$ $\left.F\left(X_{0}: X_{1}, l(\theta)\right)=0,0 \leq \theta \leq 2 \pi\right\}$ and $\widetilde{R}=\operatorname{pr}_{1}(R)$. We fix a subset $U$ of $\mathbf{P}^{1}$ which is homeomorphic to a closed disk and contains $\widetilde{R}$ in its inside.

Since we can consider $R$ as closed braid, we can naturally define homeomorphisms $\Omega_{\theta}^{\prime}: U \times\{l(0)\} \rightarrow U \times\{l(\theta)\}$ continuously along $l$ satisfying the following:

(i) $\Omega_{0}^{\prime}$ is the identity and $\Omega_{\theta}^{\prime}(p, l(0))=(p, l(\theta))$ for all $p \in \partial U$,

(ii) $\Omega_{\theta}^{\prime}$ sends the set $\left\{a_{1}^{0}, \ldots, a_{2 g+2}^{0}\right\}$ to the set $\left\{a_{1}^{\theta}, \ldots, a_{2 g+2}^{\theta}\right\}$.

We define $\Omega_{\theta}: \Gamma_{l(0)} \rightarrow \Gamma_{l(\theta)}$ as $\Omega_{\theta}(p):=\Omega_{\theta}^{\prime}(p, l(0))$ if $p \in U$ and $\Omega_{\theta}(p):=(p, l(\theta))$, otherwise. Using the map $\Omega_{\theta}$ and the defining equation of $S$ on $V(L)$, we can uniquely define the homeomorphisms $\Phi_{\theta}: f^{-1}(l(0)) \rightarrow f^{-1}(l(\theta))$ continuously along $l$ satisfying that $\left.\Omega_{\theta} \circ \Pi\right|_{f^{-1}(l(0))}=\left.\Pi\right|_{f^{-1}(l(\theta))} \circ \Phi_{\theta}$. 
Let $I$ be the analytic hyperelliptic involution of $f^{-1}(l(0))$. From $\left.\Omega_{2 \pi} \circ \Pi\right|_{f^{-1}(l(0))}=$ $\left.\Pi\right|_{f^{-1}(l(2 \pi))} \circ \Phi_{2 \pi}$ and $l(0)=l(2 \pi)$, we obtain that $\left.\Omega_{2 \pi} \circ \Pi\right|_{f^{-1}(l(0))} \circ I=\left.\Pi\right|_{f^{-1}(l(0))} \circ$ $\Phi_{2 \pi} \circ I$. On the other hand, $I$ is induced from the covering transformation of $\Pi$, we see that $\left.\Pi\right|_{f^{-1}(l(0))} \circ I=\left.\Pi\right|_{f^{-1}(l(0))}$. Then we have $\left.\Omega_{2 \pi} \circ \Pi\right|_{f^{-1}(l(0))}=\left.\Pi\right|_{f^{-1}(l(0))} \circ \Phi_{2 \pi} \circ I$. Using $\left.\Omega_{2 \pi} \circ \Pi\right|_{f^{-1}(l(0))}=\left.\Pi\right|_{f^{-1}(l(0))} \circ \Phi_{2 \pi}$ again, we see that $\left.\Pi\right|_{f^{-1}(l(0))} \circ \Phi_{2 \pi}=$ $\Pi_{f^{-1}(l(0))} \circ \Phi_{2 \pi} \circ I$. Thus we see that $I \circ \Phi_{2 \pi}=\Phi_{2 \pi} \circ I$, i.e., the monodromy of $(\tilde{f}, \widetilde{S}, \Delta)$ is hyperelliptic.

Since any hyperelliptic degeneration is bimeromorphic to a degeneration obtained by the above construction [4], we see that the monodromies of hyperelliptic degenerations are hyperelliptic. If a hyperelliptic degeneration $f: \widetilde{S} \rightarrow \Delta$ is obtained from $\Pi: S \rightarrow \mathbf{P}^{1} \times \Delta$ branched along $D$, we call $D$ the associated branch locus of $f: \widetilde{S} \rightarrow \Delta$.

Conversely, we see that the conjugacy classes of hyperelliptic periodic elements can be realized as the monodromies of hyperelliptic degenerations as follows.

Let $\tau: \Sigma_{g} \rightarrow \Sigma_{g}$ be a periodic map with period $n$ which commutes with a hyperelliptic involution $I$. Let $\langle\tau, I\rangle$ be the finite group generated by $\tau$ and $I$. By Kerckhoff's theorem [10], we see that there exist an algebraic curve $\widetilde{\Sigma}_{g}$ of genus $g$ and analytic automorphisms $\tilde{\tau}: \widetilde{\Sigma}_{g} \rightarrow \widetilde{\Sigma}_{g}, \widetilde{I}: \widetilde{\Sigma}_{g} \rightarrow \widetilde{\Sigma}_{g}$ such that $\tilde{\tau}$ and $\widetilde{I}$ are isotopic to $\tau$ and $I$, respectively. Note that $\widetilde{\Sigma}_{g}$ is a hyperelliptic curve because $\widetilde{I}$ is the hyperelliptic involution in the analytic sense. Let $\widetilde{\Sigma}_{g} \times \Delta$ be the direct product and $\langle\bar{\tau}\rangle$ the cyclic group generated by the automorphism $\bar{\tau}: \widetilde{\Sigma}_{g} \times \Delta \rightarrow \widetilde{\Sigma}_{g} \times \Delta\left((p, t) \mapsto\left(\tilde{\tau}(p), e^{2 \pi i / n} t\right)\right)$.

Let $\bar{S}$ be the nonsingular minimal model of the quotient space $\widetilde{\Sigma}_{g} \times \Delta /\langle\bar{\tau}\rangle$. Then we can naturally construct the degeneration $(f, \bar{S}, \Delta)$. From the construction of the degeneration, we see that the general fibers are hyperelliptic curves and the monodromy of the degeneration is the conjugacy class of the isotopy class of $\tau$. Then, we see that the conjugacy classes of hyperelliptic periodic elements in $\mathcal{M}_{g}$ can be realized as the monodromies of hyperelliptic degenerations.

Consequently, we see that classifying the conjugacy classes of hyperelliptic periodic elements is equal to classifying the periodic monodromies of hyperelliptic degenerations.

In [6], we described the branch loci of double coverings that give the periodic monodromies. In the following lemma, $\Delta$ is a sufficiently small disk on $\mathbf{C}$.

Lemma 1.1. Let $\mathcal{P H}_{g}$ be the set of the conjugacy classes of periodic maps in the mapping class group of genus $g$ which are realized as the monodromies of hyperelliptic degenerations. Let $\mathcal{E}_{g}$ be the set of double coverings over $\mathbf{P}^{1} \times \Delta$ whose branch loci are defined by the following equations:

(i) $\left(X_{0}-X_{1}\right) \Pi_{j=1}^{\delta}\left(X_{0}^{p}-\alpha_{j} t^{q} X_{1}^{p}\right)=0,(p \delta=2 g+1)$,

(ii) $X_{0}\left(X_{0}-X_{1}\right) \Pi_{j=1}^{\delta}\left(X_{0}^{p}-\alpha_{j} t^{q} X_{1}^{p}\right)=0,(p \delta=2 g)$,

(iii) $\Pi_{j=1}^{\delta}\left(X_{0}^{p}-\alpha_{j} t^{q} X_{1}^{p}\right)=0,(p \delta=2 g+2)$, 


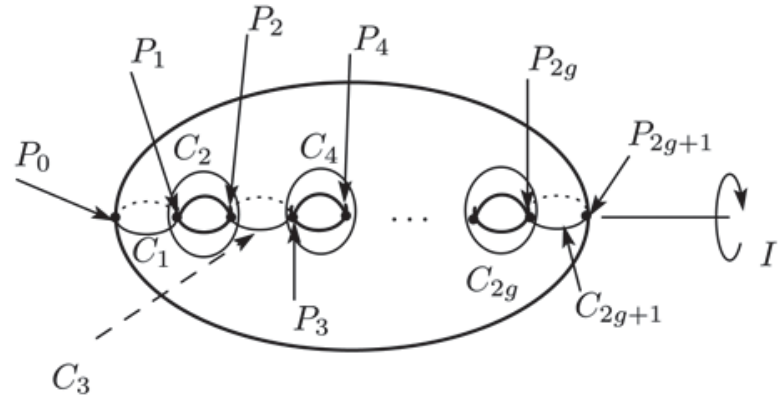

Figure 1

(iv) $t \prod_{j=1}^{\delta}\left(X_{0}^{p}-\alpha_{j} t^{q} X_{1}^{p}\right)=0,(p \delta=2 g+2)$,

(v) $\left(X_{0}-X_{1}\right) \Pi_{j=1}^{2 g+1}\left(X_{0}-\alpha_{j} t X_{1}\right)=0$,

(vi) $\Pi_{j=1}^{2 g+2}\left(X_{0}-\alpha_{j} X_{1}\right)=0$,

where $p$ and $q$ are relatively prime positive integers with $p \neq 1$ and $\left\{\alpha_{j}\right\}$ is a set of mutually distinct complex numbers. Let $\Theta: \mathcal{E}_{g} \rightarrow \mathcal{P} \mathcal{H}_{g}$ be the map which sends a double covering to its monodromy. Then $\Theta$ is surjective.

The presentation of the hyperelliptic involution by Dehn twists was obtained by Birman and Hilden [2] as follows

$$
D_{C_{1}} D_{C_{2}} \cdots D_{C_{2 g}} D_{C_{2 g+1}} D_{C_{2 g+1}} D_{C_{2 g}} \cdots D_{C_{2}} D_{C_{1}}
$$

where $\left\{C_{i}\right\}$ are simple closed curves as shown in figure 1. Since we have to clarify the relation of branch loci of double coverings and the hyperelliptic involution, we need the following

Lemma 1.2. Let $\psi_{1}: S_{1} \rightarrow \Delta$ and $\psi_{2}: S_{2} \rightarrow \Delta$ be hyperelliptic degenerations whose associated branch loci are $D$ and $D+\Gamma_{0}$, respectively. Assume that $D-\Gamma_{0}$ is not positive divisor. Let $l:[0,2 \pi] \rightarrow \Delta\left(\theta \mapsto e^{i \theta}\right)$ be a simple closed curve on $\Delta$. We denote the hyperelliptic involution of $\psi_{1}^{-1}(l(0))$ by $I_{1}$. Let $\omega_{1}$ be a monodromy homeomorphism along $l$ with the reference fiber $\psi_{1}^{-1}(l(0))$. Then the monodromy of $\psi_{2}: S_{2} \rightarrow \Delta$ is the conjugacy class of the isotopy class of $\omega_{1} \cdot I_{1}$.

Proof. Set $U_{0}:=\left\{\left(X_{0}: X_{1}\right) \in \mathbf{P}^{1} \mid X_{1} \neq 0\right\}$. We may assume that $D$ does not intersect $X_{1}=0$ by a suitable coordinates change. Let $x:=X_{0} / X_{1}$ be an affine coordinate of $U_{0}$ and $F(x, t)=0$ the defining equation of $D$ on $U_{0} \times \Delta$. Then, the defining equation of $D+\Gamma_{0}$ on $U_{0} \times \Delta$ is $t F(x, t)=0$. Let $L$ and $y$ be the line bundle satisfying $L^{\otimes 2} \simeq[D]$ and a fiber coordinate on $U_{0} \times \Delta$, respectively. Note that $S_{1}$ 
and $S_{2}$ are locally defined by the equations $y^{2}=F(x, t)$ and $y^{2}=t F(x, t)$ on $V(L)$, respectively. The morphism $\psi_{i}(i=1,2)$ is the restriction of the natural projection $L \rightarrow \Delta$ to $S_{i}(i=1,2)$. By the natural isomorphism, we identify two reference fibers $\psi_{1}^{-1}(l(0))$ and $\psi_{2}^{-1}(l(0))$.

Let $\Omega_{\theta}: \Gamma_{1} \rightarrow \Gamma_{\theta}\left(x \mapsto \Omega_{\theta}(x)\right)$ be the homeomorphism with respect to $\psi_{1}$ constructed in this section. Let $p=\left(x_{p}, 1, y_{p}\right)$ be a point on $\psi_{1}^{-1}(l(0))$. Note that it satisfies that $y_{p}^{2}=F\left(x_{p}, 1\right)$. Thus, we can construct $\Phi_{\theta}: \psi_{1}^{-1}(l(0)) \rightarrow \psi_{1}^{-1}(l(\theta))$ as $\Phi_{\theta}(p):=\left(\Omega_{\theta}\left(x_{p}\right), e^{i \theta}, \sqrt{F\left(\Omega_{\theta}\left(x_{p}\right), e^{i \theta}\right)}\right)$, where $\sqrt{F\left(\Omega_{\theta}, e^{i \theta}\right)}$ is the branch so that $\Phi_{\theta}$ is continuous along $l$. Since the defining equation of $S_{2}$ is $y^{2}=t F(x, t)$, we can define $\Phi_{\theta}^{\prime}: \psi_{2}^{-1}(l(0)) \rightarrow \psi_{2}^{-1}(l(\theta))$ as $\Phi_{\theta}^{\prime}(p):=\left(\Omega_{\theta}\left(x_{p}\right), e^{i \theta}, \sqrt{e^{i \theta} F\left(\Omega_{\theta}\left(x_{p}\right), e^{i \theta}\right)}\right)$. Thus we obtain

$$
\begin{aligned}
\Phi_{2 \pi}^{\prime}(p) & :=\left(\Omega_{2 \pi}\left(x_{p}\right), e^{2 \pi i}, \sqrt{e^{2 \pi i} F\left(\Omega_{2 \pi}\left(x_{p}\right), e^{2 \pi i}\right)}\right) \\
& =\left(\Omega_{2 \pi}\left(x_{p}\right), e^{2 \pi i},-\sqrt{F\left(\Omega_{2 \pi}\left(x_{p}\right), e^{2 \pi i}\right)}\right) \\
& =I_{1}\left(\Phi_{2 \pi}(p)\right) .
\end{aligned}
$$

Thus, we obtain the assertion.

We set $F_{1}:=\left(X_{0}-X_{1}\right)\left(X_{0}^{2 g+1}-t X_{1}^{2 g+1}\right), F_{2}:=X_{0}\left(X_{0}-X_{1}\right)\left(X_{0}^{2 g}-t X_{1}^{2 g}\right)$, $F_{3}:=X_{0}^{2 g+2}-t X_{1}^{2 g+2}, \Delta:=\{t \in \mathbf{C}|| t \mid<1\}$, and $\widetilde{\Delta}:=\{t \in \mathbf{C}|| t \mid<2\}$. Let $f_{1}: S_{1} \rightarrow \Delta, f_{2}: S_{2} \rightarrow \Delta$ and $f_{3}: S_{3} \rightarrow \widetilde{\Delta}$ be hyperelliptic degenerations whose associated branch loci are defined by $F_{1}=0, F_{2}=0$, and $F_{3}=0$, respectively. Let $\phi_{i}(i=1,2,3)$ be the isotopy class of a monodromy homeomorphism of $\left(f_{i}, S_{i}, \Delta\right)$ $(i=1,2,3)$. Let $I_{3}$ be the analytic hyperelliptic involution of the reference fiber $f_{3}^{-1}(1)$.

Lemma 1.3. Let $[\phi]$ be the conjugacy class of a hyperelliptic periodic element of $\mathcal{M}_{g}$. Then, there exists a positive integer $k$ such that $[\phi]$ is equal to the one of the following:
(a) $\left[\phi_{1}^{k}\right]$,
(b) $\left[\phi_{2}^{k}\right]$,
(c) $\left[\phi_{3}^{k}\right]$,
(d) $\left[\phi_{3}^{k} I_{3}\right]$.

Proof. For each equation in Lemma 1.1, we may put $\alpha_{j}=e^{2 \pi j i / \delta}$ without changing the monodromy. Thus, we obtain that $\Pi_{j=1}^{\delta}\left(X_{0}^{p}-\alpha_{j} t^{q} X_{1}^{p}\right)=X_{0}^{p \delta}-t^{q \delta} X_{1}^{p \delta}$. By Lemma 1.1, we see that, for each $[\phi]$, there exists a hyperelliptic degeneration $(f, S, \Delta)_{[\phi]}$ with monodromy $[\phi]$ whose associated branch locus is defined by one of the equations (i)-(vi) in Lemma 1.1.

Assume that the defining equation of the associated branch locus of $(f, S, \Delta)_{[\phi]}$ is (i), $\left(X_{0}-X_{1}\right)\left(X_{0}^{2 g+1}-t^{q \delta} X_{1}^{2 g+1}\right)=0$. Since $(f, S, \Delta)$ is obtained from $\left(f_{1}, S_{1}, \Delta\right)$ by the base change $h: \Delta \rightarrow \Delta\left(t \mapsto t^{q \delta}\right)$ of degree $q \delta$, we see that $[\phi]=\left[\phi_{1}^{q \delta}\right]$. This is the case of (a). Since the family whose associated branch locus coincides with Lemma 1.1 (v) (resp. (vi)) is obtained by the base change of $\left(f_{1}, S_{1}, \Delta\right)$ of degree $2 g+1$ (resp. $4 g+2$ ), we see that the monodromy coincides with $\left[\phi_{1}^{2 g+1}\right]$ (resp. 
$\left.\left[\phi_{1}^{4 g+2}\right]\left(=\left[\mathrm{id}_{\Sigma_{g}}\right]\right)\right)$. By the same argument, we see that the cases (ii) and (iii) in Lemma 1.1 correspond to the cases (b) and (c), respectively. By Lemma 1.2, we see that the monodromy of the families defined by $(\mathrm{d})$ is $\left[\phi_{3}^{q \delta} I_{3}\right]$.

\section{Construction of splitting families}

In this section, we give presentations of periodic monodromies $\left[\phi_{1}\right],\left[\phi_{2}\right]$, and $\left[\phi_{3}\right]$. Our method is to use the splitting deformation as follows (note that this strategy itself originated from [12]):

We "divide" the monodromy of degeneration $(f, S, \Delta)$ into Dehn twists "geometrically." Let $\Psi: \mathcal{S} \rightarrow \Delta \times \Delta^{\prime}$ be a proper flat morphism from a three dimensional complex manifold $\mathcal{S}$ to the direct product of small disks $\Delta \times \Delta^{\prime}:=\left\{(t, s) \in \mathbf{P}^{2} \mid\right.$ $\left.|t|<\varepsilon,|s|<\varepsilon^{\prime}\right\}$ whose general fiber are nonsingular algebraic curves of genus $g$. We set $\Delta_{s}:=\Delta \times\{s\}, \mathcal{S}_{s}:=\Psi^{-1}\left(\Delta_{s}\right)$ and $\Psi_{s}:=\left.\Psi\right|_{\mathcal{S}_{s}}$. If $\mathcal{S}_{0}=S, \Delta_{0}=\Delta, \Psi_{0}=f$, and each family $\left(\Psi_{s}, \mathcal{S}_{s}, \Delta_{s}\right)\left(s \in \Delta^{\prime} \backslash\{0\}\right)$ has at least two critical points, we call the triple $\left(\Psi, \mathcal{S}, \Delta \times \Delta^{\prime}\right)$ a splitting family of $(f, S, \Delta)$. Fix a point $s \in \Delta^{\prime} \backslash\{0\}$. Let $l_{0}:[0,2 \pi] \rightarrow \Delta \times \Delta^{\prime}$ be a path on $\Delta \times \Delta^{\prime}$ connecting a point on $\Delta_{0}$ with a point $\Delta_{s}$ satisfying that $\Psi^{-1}\left(l_{0}(\theta)\right)$ is nonsingular for all $\theta(\in[0,2 \pi])$. Let $l_{1}, l_{2}, \ldots, l_{m}$ be simple closed curves on $\Delta_{s}$ beginning at $l_{0}(2 \pi)$ such that each curves rounds only one critical point and the curve $l_{1} \circ l_{2} \circ \cdots \circ l_{m}$ is homotopic to a simple closed curve rounding all critical points on $\Delta_{s}$ with counter clockwise orientation. Since $l_{0} \circ l_{1} \circ \cdots \circ l_{m} \circ l_{0}^{-1}$ is homotopic to a simple closed curve $l$ on $\Delta_{0}$ rounding the critical point, we see that $\left[\phi_{f}^{l}\right]=\left[\phi_{\Psi_{s}}^{l_{1}} \circ \ldots \circ \phi_{\Psi_{s}}^{l_{m}}\right]$. If $\left(\Psi_{s}, \mathcal{S}_{s}, \Delta_{s}\right)$ is a Lefschetz fibration, we obtain the presentation of monodromy of $(f, S, \Delta)$ by Dehn twist as $\left[D_{1} D_{2} \cdots D_{m}\right]$, where $D_{i}$ is the Dehn twist that is the isotopy class of $\phi_{\Psi_{s}}^{l_{i}}$.

Then, in this section, for each hyperelliptic degeneration with monodromy $\left[\phi_{j}\right]$, we construct a splitting family and observe the vanishing cycles and a composition rule of local monodromies.

Theorem 2.1. Let $C_{1}, C_{2}, \ldots, C_{2 g+1}$ be simple closed curves in $\Sigma_{g}$ as is shown in figure 1. Then,

(i) $\left[\phi_{1}\right]=\left[D_{C_{1}} D_{C_{2}} \cdots D_{C_{2 g}}\right]$,

(ii) $\left[\phi_{2}\right]=\left[D_{C_{1}} D_{C_{2}} \cdots D_{C_{2 g}} D_{C_{2 g}}\right]$,

(iii) $\left[\phi_{3}\right]=\left[D_{C_{1}} D_{C_{2}} \cdots D_{C_{2 g}} D_{C_{2 g+1}}\right]$.

Proof. The case (iii) has already been proved in [7]. We prove (i).

Let $\left(X_{0}: X_{1}\right)$ be a homogeneous coordinates of $\mathbf{P}^{1}$ and $(t, s)$ a coordinates of $\Delta \times \Delta^{\prime}:=\{(t, s) \in \mathbf{C} \times \mathbf{C}|| t|<8 g| s \mid,<1+\varepsilon\}$, where $\varepsilon$ is a positive number. Let $\left(\overline{f_{1}}, \overline{S_{1}}, \Delta\right)$ be the hyperelliptic degeneration whose associated branch locus is $\overline{F_{1}}:=\left(X_{0}-4 g X_{1}\right)\left(X_{0}^{2 g+1}+t X_{1}^{2 g+1}\right)=0$. Since we can obtain $\overline{F_{1}}$ from $F_{1}$ by a suitable coordinates change, we see that the monodromy of $\left(\overline{f_{1}}, \overline{S_{1}}, \Delta\right)$ is $\left[\phi_{1}\right]$. 


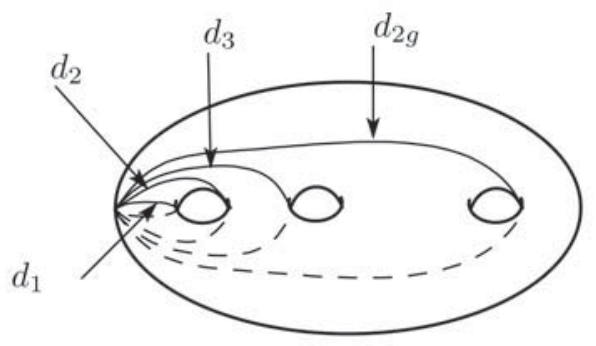

Figure 2

We construct a splitting family of $\left(\overline{f_{1}}, \overline{S_{1}}, \Delta\right)$ as follows. Set $\varepsilon=1$. Let $D$ be the divisor on $\mathbf{P}^{1} \times \Delta \times \Delta^{\prime}$ defined by the equation $H\left(X_{0}: X_{1}, t, s\right):=\left(X_{0}-4 g X_{1}\right)\left(X_{0}^{2 g+1}\right.$ $\left.(2 g+1) s X_{0} X_{1}^{2 g}+t X_{1}^{2 g+1}\right)=0$. Since the associated line bundle $[D]$ is an even bundle on $\mathbf{P}^{1} \times \Delta \times \Delta^{\prime}$, we can construct a double covering $\Theta: \mathcal{S} \rightarrow \mathbf{P}^{1} \times \Delta \times \Delta^{\prime}$ branched along $D$. Since $D$ is nonsingular, $\mathcal{S}$ is nonsingular variety of dimension three. Let $p_{23}: \mathbf{P}^{1} \times \Delta \times \Delta^{\prime} \rightarrow \Delta \times \Delta^{\prime}$ be the natural projection and set $\Psi:=p_{23} \circ \Theta: \mathcal{S} \rightarrow \Delta \times \Delta^{\prime}$. Since $H\left(X_{0}: X_{1}, t, 0\right)=\bar{F}_{1}, \Psi_{0}=\overline{f_{1}}$ and $\mathcal{S}_{1}=\overline{S_{1}}$, we see that $\left(\Psi, \mathcal{S}, \Delta \times \Delta^{\prime}\right)$ is a splitting family of $\left(\overline{f_{1}}, \overline{S_{1}}, \Delta\right)$. Moreover, by easy calculations, we see that $\left(\Psi_{s}, \mathcal{S}_{s}, \Delta_{s}\right)$ $\left(s \in \Delta^{\prime} \backslash\{0\}\right)$ is of Lefschetz type with $2 g$ singular fibers.

For simplicity, we observe the monodromy homeomorphisms of the family $\Psi_{1}: \mathcal{S}_{1} \rightarrow \Delta_{1}$. Set $U:=\left\{\left(X_{0}: X_{1}, t\right) \in \mathbf{P}^{1} \times \Delta_{1} \mid X_{1} \neq 0\right\}, x:=X_{0} / X_{1}$, $h(x, t):=H(x: 1, t, 1)$, and $\eta:=e^{2 \pi i / 2 g}$. It is sufficient to observe $\mathcal{S}_{1}$ over $U$ because $D$ does not intersect the divisor defined by $X_{1}=0$. Since $\Psi_{1}^{-1}(p)$ is non-singular if and only if $h(x, t)=0$ has $2 g+2$ distinct roots, the critical points of the family are $t=2 g \eta^{j}(j=0,1, \ldots, 2 g-1)$.

Let $L_{j}(j=0,1, \ldots, 2 g-1)$ be the segment on $\Delta_{1}$ which connects the origin and $t=2 g \eta^{j}(j=0,1, \ldots, 2 g-1)$ and $l_{j}$ a simple closed curve whose initial point is the origin and rounding only one critical point $t=2 g \eta^{j}$ with counter clockwise orientation. We take $\Psi_{1}^{-1}(0)$ as a reference fiber. Note that $\Psi_{1}^{-1}(0)$ is locally defined by $y^{2}=(x-4 g)\left(x^{2 g+1}-(2 g+1) x\right)$. We denote the positive real roots of the equation $x^{2 g+1}-(2 g+1) x=0$ by $\alpha$. When $t$ moves on $L_{0}$ from the origin to $t=2 g$, the two real roots of $x^{2 g+1}-(2 g+1) x+t=0$ become near and finally overlap each other. Then, we see that the simple closed curve on $\Psi_{1}^{-1}(0)$ which is the lift of the segment connecting the two real roots $x=0$ and $x=\alpha$ of the equation $x^{2 g+1}-(2 g+1) x=0$ is the vanishing cycle. From this, we see that the monodromy homeomorphism around $t=2 g$ is the Dehn twist in this vanishing cycle [9].

In the case of when $t$ moves on $L_{j}$, we see that the vanishing cycle is the lift of the segment which connects two roots $x=0$ and $x=\alpha \eta^{j}$ to $\Psi^{-1}(0)$. We describe the vanishing cycles associated to $L_{j}$ as $d_{j+1}$ in figure 2 . In figure $1, P_{j}(j=1,2, \ldots, 2 g)$ is the point on $\Psi_{1}^{-1}(0)$ which is the lift of the point $x=\alpha \eta^{j-1}(j=1,2, \ldots, 2 g)$. The 
points $P_{0}$ and $P_{2 g+1}$ are the points which are the lifts of the points $x=0$ and $x=4 g$, respectively. Then, calculating the monodromy along the curve $l_{0} \circ l_{1} \circ \cdots \circ l_{2 g-1}$, we see that $\left[\phi_{1}\right]=\left[D_{d_{1}} D_{d_{2}} \cdots D_{d_{2 g}}\right]$. Since

$$
D_{d_{j}}=D_{C_{j}}^{-1} D_{C_{j-1}}^{-1} \cdots D_{C_{2}}^{-1} D_{C_{1}} D_{C_{2}} \cdots D_{C_{j-1}} D_{C_{j}},
$$

we see that $D_{d_{1}} D_{d_{2}} \cdots D_{d_{2 g}}=D_{C_{1}} D_{C_{2}} \cdots D_{C_{2 g}}$ by easy calculation (see [7]). Then we obtain the assertion (i).

We prove (ii). Since we obtain $\overline{F_{2}}:=X_{0}\left(X_{0}-4 g X_{1}\right)\left(X_{0}^{2 g}+t X_{1}^{2 g}\right)$ from $F_{2}$ by a suitable coordinates change, we see that the monodromy of the hyperelliptic degeneration $\left(\overline{f_{2}}, \overline{S_{2}}, \Delta\right)$ with the associated branch locus $\bar{F}_{2}=0$ is $\left[\phi_{2}\right]$.

Fix a positive number $\varepsilon$ satisfying that $8 g>2 g(1+\varepsilon)^{2}+(1+\varepsilon)^{2 g}$. We construct the double covering $\alpha: \mathcal{M} \rightarrow \mathbf{P}^{1} \times \Delta \times \Delta^{\prime}$ branched along the divisor defined by $J\left(X_{0}: X_{1}, t, s\right):=\left(X_{0}+s X_{1}\right)\left(X_{0}-4 g X_{1}\right)\left(X_{0}^{2 g}-2 g s X_{0} X_{1}^{2 g-1}+t X_{1}^{2 g}\right)=0$. Set $\beta:=p_{23} \circ \alpha: \mathcal{M} \rightarrow \Delta \times \Delta^{\prime}$. The branch locus of $\alpha$ is singular along $\operatorname{Sing}(J):=$ $\left\{\left(-s: 1,-2 g s^{2}-s^{2 g}, s\right) \mid s \in \Delta^{\prime}\right\}$. Since, for each $s$, the singular point of $J\left(X_{0}: X_{1}, t, s\right)=0$ is an ordinary double point, we see that the singular point on $\mathcal{M}_{s}$ is a rational double point of Type $A_{1}$. Since $\mathcal{M}$ is the total space of the equisingular deformation of the singular point of $\mathcal{M}_{0}$, we obtain the splitting family of $\left(\overline{f_{2}}, \overline{S_{2}}, \Delta\right)$ by the blowing-up along $\alpha^{-1}(\operatorname{Sing}(J))$.

We observe the family of curves $\beta_{1}: \mathcal{M}_{1} \rightarrow \Delta_{1}$ which has a singular points over $\beta_{1}^{-1}(-2 g-1)$. It is sufficient to observe $\mathcal{M}_{1}$ over $U$ because the divisor $J\left(X_{0}\right.$ : $\left.X_{1}, t, 1\right)=0$ does not intersect the divisor $X_{1}=0$. Set $J(x, t):=J(x: 1, t, 1)$ and $\xi:=e^{2 \pi i /(2 g-1)}$. Then, the equation $J(x, t)=0$ has a multiple root when $t=(2 g-1) \xi^{j}$ $(j=0,1, \ldots, 2 g-2)$ and $t=-2 g-1$. By easy calculation, we see that each singular fiber has only one node as singularity and the singular point of Type $A_{1}$ is on the node of $\beta_{1}^{-1}(-2 g-1)$.

As similar in the case of the proof of (i), we denote by $L_{j}(j=0,1, \cdots, 2 g-2)$ the segment on $\Delta_{1}$ connecting the origin and the point $t=(2 g-1) \xi^{j}(j=0,1, \cdots, 2 g-2)$. We denote by $\bar{L}$ the segment connecting the origin and $t=-2 g-1$. Let $l_{j}$ and $\bar{l}$ be simple closed curves with its initial point at the origin and rounding only one critical point $t=(2 g-1) \xi^{j}$ and $t=-2 g-1$, respectively. We take $\beta_{1}^{-1}(0)$ as the reference fiber. The nonsingular fiber $\beta_{1}^{-1}(0)$ is locally defined by $y^{2}=(x+1)(x-4 g)\left(x^{2 g}-2 g x\right)$.

Let $\sigma$ be the positive real root of the equation $x^{2 g}-2 g x=0$. By the same observation in the case (i), we see that the lift on $\beta_{1}^{-1}(0)$ of the segment connecting the points $x=0$ and $x=\sigma \xi^{j}$ is the vanishing cycle associated to $L_{j}$.

Next, we determine the monodromy around $\beta_{1}^{-1}(-2 g-1)$. Assume that $t$ is a real number. Let $z_{1}(t)$ be the real root of the equation of $x^{2 g}-2 g x+t=0$ which is less than the other real root of the equation. When $t$ moves on $\bar{L}$ from the origin to $t=-2 g-1, z_{1}(t)$ moves from 0 to -1 . Then the vanishing cycle with respect to $\bar{L}$ is the lift of the segment connecting the points $x=0$ and $x=-1$. Then, we see that the monodromy is the two times Dehn twists on this vanishing cycle because the singular point on the node of $\beta_{1}^{-1}(-2 g-1)$ is of type $A_{1}$. 


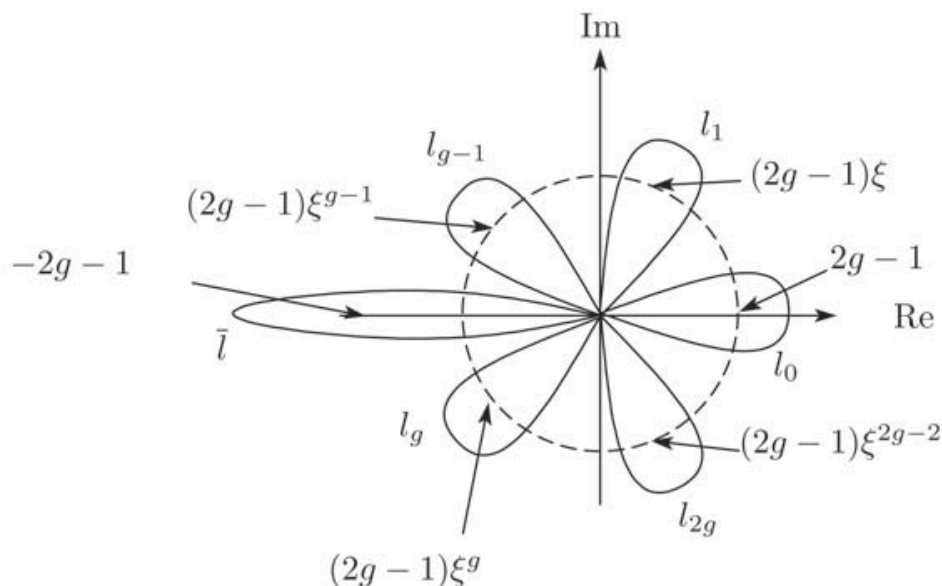

Figure 3

We explain the configurations of the vanishing cycles, branch points of $\beta_{1}^{-1}(0) \rightarrow \mathbf{P}^{1}$, and the critical points of $\beta_{1}$. In figure 1 , the points $P_{0}, P_{2 g}$, and $P_{2 g+1}$ are the lifts of the points $x=0, x=-1$, and $x=4 g$, respectively. The points $P_{1}, \ldots, P_{2 g-1}$ are the lifts of the points of $x=\sigma \xi^{g}, \ldots, \sigma \xi^{2 g-2}, \sigma, \sigma \xi, \ldots, \sigma \xi^{g-1}$, respectively. In figure 3 , we describe the positions of the critical points, $l_{j}$ and $\bar{l}$. As the same observations in the proof of (i), we see that the vanishing cycles of the monodromies along $l_{g}, \ldots, l_{2 g-2}, l_{0}, l_{1}, \ldots l_{g-1}, \bar{l}$ are $d_{1}, d_{2}, \ldots, d_{2 g-1}, d_{2 g}$, respectively.

Calculating the monodromies along the curve $l_{g} \circ l_{g+1} \circ \cdots \circ l_{2 g-2} \circ l_{0} \circ l_{1} \circ l_{2} \circ$ $\cdots \circ l_{g-1} \circ \bar{l}$ (see figure 3), we obtain that $\left[\phi_{2}\right]=\left[D_{d_{1}} \cdots D_{d_{2 g}} D_{d_{2 g}}\right]$. By the same calculation in the case of (i), we obtain $\left[\phi_{2}\right]=\left[D_{C_{1}} D_{C_{2}} \cdots D_{C_{2 g}} D_{C_{2 g}}\right]$.

\section{Method for Making the List of Presentations}

In this section, we first review the configurations of the singular fibers of degenerations with periodic monodromies. Next, we introduce the method for classifying the hyperelliptic monodromies with their periods and total valencies. We also introduce the method for obtaining a presentation for each periodic monodromy. At the end of this section, we add the list of periodic monodromies in the case where $g=2$ and 3 . Using this list, we can easily obtain presentations by Dehn twists of them.

By Nielsen's theorem, the conjugacy class of a periodic element $\phi$ is characterized by two invariants, the period and the total valency (see [14] or [1]). The period of $\phi$ is the smallest positive integer $n$ satisfying $\phi^{n}=\mathrm{id}_{\Sigma_{g}}$ and the total valency is expressed as the formal sum of fractional numbers as $n_{1} / m_{1}+\cdots+n_{k} / m_{k}$.

Let $(f, S, \Delta)$ be a degeneration with the monodromy $[\phi]$ whose period and total 
valency are as above. Assume that $(f, S, \Delta)$ is normally minimal, i.e., the reduced scheme of the singular fiber has only nodes as singularities and any $(-1)$ curve intersects the other components at at least three points. The normally minimal model exists uniquely among the bimeromorphic equivalence class of any degeneration. Then, we see that the configuration of the singular fiber is as follows $([13])$ :

Since $\phi$ is a periodic map, we can consider the quotient space $\Sigma_{g} /\langle\phi\rangle$ of $\Sigma_{g}$ by the group generated by $\phi$. Let $g^{\prime}$ be the genus of the quotient. Let $\left(n_{i}^{(0)}, n_{i}^{(1)}, \ldots, n_{i}^{\left(s_{i}\right)}\right)$ be the sequence of positive integers which satisfies the following:

(i) $n_{i}^{(0)}=n$ and $n_{i}^{(1)}=n n_{i} / m_{i}$,

(ii) $n_{i}^{(j)}$ is the smallest positive integer such that $n_{i}^{(j)}+n_{i}^{(j-2)}$ is a multiple of $n_{i}^{(j-1)}$.

Then, the singular fiber of $(f, S, \Delta)$ can be written down as $X_{[\phi]}=n \Theta+$ $\sum_{i=1}^{k} \Sigma_{j=1}^{s_{i}} n_{i}^{(j)} E_{i}^{j}$ which satisfies the following conditions:

(i) $\Theta$ is a smooth curve of genus $g^{\prime}$ and $E_{i}^{j}$ are nonsingular rational curves.

(ii) $\Theta \cdot E_{i}^{1}=E_{i}^{1} E_{i}^{2}=\cdots=E_{i}^{s_{i}-1} E_{i}^{s_{i}}=1, E_{i}^{j} E_{i}^{j^{\prime}}=0\left(\left|j-j^{\prime}\right| \geq 2\right), E_{i_{1}}^{j} E_{i_{2}}^{j^{\prime}}=0$ $\left(i_{1} \neq i_{2}\right)$.

Conversely, the monodromy of a degeneration with above singular fiber is periodic with the period $n$ and the total valency $n_{1} / m_{1}+\cdots+n_{k} / m_{k}$. Thus, we can calculate the period and the total valency of the monodromy of a degeneration from the configuration of the singular fiber of it.

Remark 3.1. From the configuration of the singular fiber of $\left(\overline{f_{1}}, \overline{S_{1}}, \Delta\right)$, we see that the monodromy $\left[\phi_{1}\right]$ is a periodic with the period $4 g+2$ and the total valency $1 /(4 g+$ $2)+g /(2 g+1)+1 / 2$. We also see that the period and the total valency of $\left[\phi_{2}\right]$ are $4 g$ and $1 / 4 g+(2 g-1) / 4 g+1 / 2$, respectively. The period and the total valency of $\left[\phi_{3}\right]$ are $2 g+2$ and $1 /(2 g+2)+1 /(2 g+2)+g /(g+1)$.

Using the above facts and Lemma 1.3 and Theorem 2.1, we explain a method of obtaining the list of the periods and the total valencies of hyperelliptic periodic monodromies and their presentations by Dehn twists in any genus $g$.

Let $k_{1}, k_{2}, k_{3}$, and $k_{4}$ be integers satisfying that $1 \leq k_{1} \leq 4 g+2,1 \leq k_{2} \leq 4 g$ and $1 \leq k_{3}, k_{4} \leq 2 g+2$. Let $S_{1, k_{1}}, S_{2, k_{2}}, S_{3, k_{3}}$ and $S_{4, k_{4}}$ be normally minimal hyperelliptic degenerations whose associated branch locus are defined by $F_{1}\left(X_{0}: X_{1}, t^{k_{1}}\right)=0$, $F_{2}\left(X_{0}: X_{1}, t^{k_{2}}\right)=0, F_{3}\left(X_{0}: X_{1}, t^{k_{3}}\right)=0$ and $t F_{3}\left(X_{0}: X_{1}, t^{k_{3}}\right)=0$, respectively. By Lemma 1.3, we see that the monodromies of $S_{i, k_{i}}(i=1,2,3,4)$ are $\left[\phi_{i}^{k_{i}}\right](i=1,2,3)$ and $\left[\phi_{4}^{k_{4}}\right]:=\left[\left(\phi_{3}\right)^{k_{4}} I\right]$, respectively. Thus the method for making the list is as follows:

(i) For each $\left[\phi_{i}^{k_{i}}\right](i=1,2,3,4)$, we calculate the period and the total valency of it from the configuration of the singular fiber of $S_{i, k_{i}}(i=1,2,3,4)$. 
(ii) For each $\left[\phi_{i}^{k_{i}}\right](i=1,2,3,4)$, using Theorem 2.1 and the presentation of the hyperelliptic involution obtained by Birman and Hilden [2], attach the presentation by Dehn twists.

Note that we can easily calculate the configuration of the singular fiber of $S_{i, k_{i}}$ $(i=1,2,3,4)$ using the canonical resolution of double coverings [5].

For example, calculating the singular fiber of normally minimal model of $f$ : $S_{4,2} \rightarrow \Delta$ in the case where $g=2$, we see that the monodromy of the degeneration is $\left[\phi_{3}^{2} I\right]$ with period 6 and the valency $2 / 3+1 / 3+1 / 2+1 / 2$. We write it as $\phi_{3}^{2} I=$ $(6,2 / 3+1 / 3+1 / 2+1 / 2)$. Thus, by Theorem 2.1 and the presentation of $I$, we obtain a presentation of the periodic monodromy with the period 6 and the valency $2 / 3+1 / 3+1 / 2+1 / 2$ as

$$
\left[\phi_{3}^{2} I\right]=\left[\left(D_{C_{1}} D_{C_{2}} D_{C_{3}} D_{C_{4}} D_{C_{5}}\right)^{2} D_{C_{1}} D_{C_{2}} D_{C_{3}} D_{C_{4}} D_{C_{5}} D_{C_{5}} D_{C_{4}} D_{C_{3}} D_{C_{2}} D_{C_{1}}\right] .
$$

Since writing down of presentations of each monodromies is easy, we omit it and we make the list of the periods and the total valencies for each $\left[\phi_{i}^{k_{i}}\right]$ in the case where $g=2$ and 3 .

In the following lists, we denote the valency 0 when the canonical projection $\Sigma_{g} \rightarrow \Sigma /\langle\phi\rangle$ is unramified.

\section{List in the case where $g=2$}

$\phi_{1}=(10,1 / 10+2 / 5+1 / 2), \phi_{1}^{2}=(5,1 / 5+2 / 5+2 / 5), \phi_{1}^{3}=(10,7 / 10+4 / 5+1 / 2)$, $\phi_{1}^{4}=(5,1 / 5+1 / 5+3 / 5), \phi_{1}^{6}=(5,2 / 5+4 / 5+4 / 5), \phi_{1}^{7}=(10,3 / 10+1 / 5+1 / 2)$, $\phi_{1}^{8}=(5,3 / 5+3 / 5+4 / 5), \phi_{1}^{9}=(10,9 / 10+3 / 5+1 / 2), \phi_{2}=(8,1 / 8+3 / 8+1 / 2)$, $\phi_{2}^{2}=(4,1 / 4+3 / 4+1 / 2+1 / 2), \phi_{2}^{5}=(8,5 / 8+7 / 8+1 / 2), \phi_{3}=(6,1 / 6+1 / 6+2 / 3)$, $\phi_{3}^{2}=(3,1 / 3+1 / 3+2 / 3+2 / 3), \phi_{3}^{3}=(2,1 / 2+1 / 2), \phi_{3}^{5}=(6,5 / 6+5 / 6+1 / 3)$, $\phi_{3}^{2} I=(6,2 / 3+1 / 3+1 / 2+1 / 2), I=(2,1 / 2+1 / 2+1 / 2+1 / 2+1 / 2+1 / 2)$.

\section{List in the case where $g=3$}

$\phi_{1}=(14,1 / 14+3 / 7+1 / 2), \phi_{1}^{2}=(7,1 / 7+3 / 7+3 / 7), \phi_{1}^{3}=(14,5 / 14+1 / 7+1 / 2)$, $\phi_{1}^{4}=(7,4 / 7+5 / 7+5 / 7), \phi_{1}^{5}=(14,3 / 14+2 / 7+1 / 2), \phi_{1}^{6}=(7,5 / 7+1 / 7+1 / 7)$, $\phi_{1}^{8}=(7,2 / 7+6 / 7+6 / 7), \phi_{1}^{9}=(14,11 / 14+5 / 7+1 / 2), \phi_{1}^{10}=(7,3 / 7+2 / 7+2 / 7)$, $\phi_{1}^{11}=(14,9 / 14+6 / 7+1 / 2), \phi_{1}^{12}=(7,6 / 7+4 / 7+4 / 7), \phi_{1}^{13}=(14,13 / 14+4 / 7+1 / 2)$, $\phi_{2}=(12,1 / 12+5 / 12+1 / 2), \phi_{2}^{2}=(6,1 / 6+5 / 6+1 / 2+1 / 2), \phi_{2}^{3}=(4,1 / 4+$ $1 / 4+1 / 2+1 / 2+1 / 2), \phi_{2}^{4}=(3,1 / 3+2 / 3), \phi_{2}^{7}=(12,7 / 12+11 / 12+1 / 2), \phi_{2}^{9}=$ $(4,3 / 4+3 / 4+1 / 2+1 / 2+1 / 2), \phi_{3}=(8,1 / 8+1 / 8+3 / 4), \phi_{3}^{2}=(4,1 / 4+1 / 4+3 / 4+3 / 4)$, $\phi_{3}^{3}=(8,3 / 8+3 / 8+1 / 4), \phi_{3}^{4}=(2,1 / 2+1 / 2+1 / 2+1 / 2), \phi_{3}^{5}=(8,5 / 8+5 / 8+3 / 4)$, $\phi_{3}^{7}=(8,7 / 8+7 / 8+1 / 4), \phi_{3}^{2} I=(4,1 / 2+1 / 2), \phi_{3}^{4} I=(2,0), I=(2,1 / 2+1 / 2+1 / 2+$ $1 / 2+1 / 2+1 / 2+1 / 2+1 / 2)$. 
Acknowledgements. The author wishes to express his special thanks to Professors Yukio Matsumoto, Tadashi Ashikaga, Kazuhiro Konno, Susumu Hirose, Yuichi Yamada, Shigeru Takamura, Masaharu Ishikawa, Hirotaka Ishida, Hajime Kaji, Takeshi Kajiwara, and Nobuo Hara for useful advice and encouragement.

He also would like to thank the referee for his or her careful reading and many useful comments.

\section{References}

[1] T. Ashikaga and M. Ishizaka, Classification of degenerations of curves of genus three via Matsumoto-Montesinos' theorem, Tohoku Math. J. (2) 54 (2002), no. 2, 195-226.

[2] J. S. Birman and H. M. Hilden, On the mapping class groups of closed surfaces as covering spaces, Advances in the Theory of Riemann Surfaces (Stony Brook, N.Y., 1969), Ann. of Math. Studies, vol. 66, Princeton Univ. Press, Princeton, N.J., 1971, pp. 81-115.

[3] P. Deligne and N. Katz (eds.), Groupes de monodromie en géométrie algébrique, II, Lecture Notes in Mathematics, vol. 340, Springer-Verlag, Berlin, 1973. Séminaire de Géométrie Algébrique du Bois-Marie, (1967-1969).

[4] E. Horikawa, On algebraic surfaces with pencils of curves of genus 2, Complex analysis and algebraic geometry: A volume dedicated to K. Kodaira, Iwanami Shoten, Tokyo, 1977, pp. 79-90.

[5] _ On deformations of quintic surfaces, Invent. Math. 31 (1975), 43-85.

[6] M. Ishizaka, Classification of the periodic monodromies of hyperelliptic families, Nagoya Math. J. 174 (2004), 187-199.

[7]_- One parameter families of Riemann surfaces and presentations of elements of mapping class group by Dehn twists, J. Math. Soc. Japan 58 (2006), no. 2, 585-594.

[8] T. Ito, Splitting of singular fibers in certain holomorphic fibrations, J. Math. Sci. Univ. Tokyo 9 (2002), no. 3, 425-480.

[9] A. Kas, On the handlebody decomposition associated to a Lefschetz fibration, Pacific J. Math. 89 (1980), no. 1, 89-104.

[10] S. P. Kerckhoff, The Nielsen realization problem, Ann. of Math. (2) 117 (1983), no. 2, 235-265.

[11] W. B. R. Lickorish, A representation of orientable combinatorial 3-manifolds, Ann. of Math. (2) 76 (1962), 531-540.

[12] Y. Matsumoto, Lefschetz fibrations of genus two: A topological approach, 37th Taniguchi Symposium: Topology and Teichmüller Spaces (Katinkulta, 1995), Kojima et al. (eds.), World Sci. Publ., River Edge, NJ, 1996, pp. 123-148.

[13] Y. Matsumoto and J. M. Montesinos-Amilibia, Pseudo-periodic maps and degeneration of Riemann surfaces, I, II, Univ. of Tokyo and Univ. Complutense de Madrid, 1991/1992, preprints.

[14] J. Nielsen, Die Structur periodischer Transformationen von Flächen, Mat.-Fys. Medd. Danske Vid. Selsk. 15 (1937); English transl. in Collected Papers 2, Birkhäuser (1986). 